\title{
Fertility preservation in young cancer patients as a springboard to address the needs of this unique population
}

\author{
Herbert HF Loong *, FHKCP, FHKAM (Medicine) \\ Department of Clinical Oncology, The Chinese University of Hong Kong; Adult Sarcoma Multidisciplinary Tumour Board, \\ Prince of Wales Hospital; Shatin, Hong Kong \\ *Corresponding author: h_loong@clo.cuhk.edu.hk
}

Hong Kong Med J 2017;23:552-3 DOI: 10.12809/hkmj175072

Treatment outcomes for patients with cancer have improved greatly, in part due to more aggressive forms of systemic treatments. Such treatments, however, can compromise fertility and this has precipitated a growing focus on fertility issues within the oncology community. International guidelines on fertility preservation in cancer patients recommend that physicians discuss, as early as possible, with all patients of reproductive age their risk of infertility from the disease and/or treatment and their interest in having children after cancer, and help with informed decisions about fertility preservation. ${ }^{1,2}$ A local study performed in a major teaching hospital reported that up to $32 \%$ of male cancer patients encountered deterioration of semen parameters after gonadotoxic treatments. ${ }^{3}$ The thought of the possibility or actual prior experience of treatment-related infertility can lead to psychological stress. ${ }^{4,5}$ Patients prefer maintaining their fertility and future reproductive function at the time of cancer diagnosis. ${ }^{6}$ Fertility concerns may also affect the decision to pursue treatment. ${ }^{7-9}$ As recommended by the American Society of Clinical Oncology ${ }^{2}$ and the European Society for Medical Oncology, ${ }^{1}$ sperm cryopreservation and embryo/oocyte cryopreservation are standard strategies for fertility preservation in male and female patients, respectively. Other strategies, which include pharmacological protection of the gonads and gonadal tissue cryopreservation, are currently considered experimental. Whilst these guidelines and recommendations are readily accessible, the 'bottom-line' of whether a suitable patient is referred for fertility preservation is entirely dependent on the treating physicians' awareness and understanding as well as the local availability of fertility-preservation techniques.

In the article that accompanies this editorial, Chung et $\mathrm{al}^{10}$ present the results of a cross-sectional paper-based survey that assessed the awareness of, attitude towards, and knowledge about fertility preservation among 167 clinicians of various clinical specialties in Hong Kong. Specialists in General Surgery, Paediatrics, Clinical and Medical Oncology, and Haematology and Haematological
Oncology were included. This is the first such study ever reported from the territory. Obstetrics and Gynaecology (O\&G) specialists were also included in the survey and accounted for the largest proportion of respondents by specialty (40.7\%). A limitation of this study, however, was that all respondents were specialists working in the public sector, as the communications directory of the Hospital Authority was used to identify potential subjects. The prior referral experiences of the respondents might be different to all $O \& G$ specialists in our locality, as there is currently no publicly funded fertility centre in Hong Kong.

Results of this study ${ }^{10}$ were surprising, to say the least. Without going into the specifics of different types of fertility preservation and their respective indications, less than half of the respondents (45.6\%) reported being 'aware of fertility preservation' Specialists in O\&G fared no better in this regard with only half $(50.7 \%)$ of the respondents reporting themselves as being aware. As expected, O\&G specialists were more aware of fertility-preservation techniques in females such as oocyte- and embryofreezing as well as ovarian tissue freezing, than their non-O\&G counterparts. Interestingly, when respondents were further asked about individual fertility-preservation procedures, an increased awareness was found. In fact, a higher percentage of the same O\&G specialists in this study reported to be familiar with "all of the above" fertility-preservation techniques previously itemised, compared with being 'aware of' fertility preservation per se $(63.6 \%$ vs $50.7 \%$ ). These findings highlight a possible diversity of understanding within our medical community of what constitutes fertility preservation. Moreover, even if knowledge is indeed improved, suitable patients may still not be able to receive appropriate counselling and care, as only a little more than half (55\%) of all respondents were aware that there are dedicated clinics and specialists who would be willing to accept referrals for fertility preservation. On a more encouraging note, an overwhelming majority of respondents (97\%) felt that at least a dedicated clinic or fertility preservation centre is necessary in Hong Kong, and over half felt at least 
two centres are required to cater for both private and public patients. This study highlighted a gap in understanding among the medical community and a lack of currently available resources for fertility preservation that must be overcome if we are to truly provide this service effectively.

In general, risks of treatment-related infertility have been described previously by various groups. A recently published modified consensus ${ }^{4,11}$ divided systemic anti-cancer therapies and radiation therapy of specific doses to gonadal sites into five different risk categories, namely: (i) high risk, corresponding to $>80 \%$ risk of permanent amenorrhoea in women and prolonged azoospermia in men; (ii) intermediate risk (40\%-60\% risk of permanent amenorrhoea in women and likelihood of azoospermia in men when given with other sterilising agents); (iii) low risk $(<20 \%$ risk of permanent amenorrhoea in women and only temporary reduction in sperm counts in men); (iv) very low or no risk of permanent amenorrhoea in women and temporary reduction in sperm count in men; and (v) unknown risk of permanent amenorrhoea in women and effect on sperm production in men. It is important to note that the gonadotoxic effects of newer targeted therapies such as tyrosine-kinase inhibitors and monoclonal antibodies have not been studied in detail. The impact of these agents on a patient's subsequent fertility has also not been well described. Whilst data are now gradually emerging, there is a need for the oncology community to study the impact of these newer agents on fertility, especially since they have now become the cornerstone of effective anti-cancer treatment. A possible approach may be to analyse large population-based health and cancer registries, and cross-reference individuals who may have received these agents with subsequent successful child-bearing or birth, either through natural or assisted means.

Moving forward, from a societal perspective, it is impractical to educate all clinicians of various specialties about the latest advancements and techniques of fertility preservation. This is also not necessary. What may be a more reasonable approach is for physicians, especially oncologists and haematologists who administer gonadotoxic chemotherapies, to become more diligent in recognising the fertility-preservation needs and concerns of 'younger' oncology patients, and to have ready access to referrals and consultative services that fertility specialists can provide. Fertility specialists should also be made more aware of both the improved treatment outcomes as well as their potential toxicities. This should not only be limited to toxicities associated with fertility, but with other physical side-effects as well as potential socio-economic burdens that newer anti-cancer treatments entail.

Physical, psychosocial, and economic impacts of cancer care, as well as the natural history of the disease, will likely affect a patient's decision about whether to pursue fertility preservation. Younger patients who are often in the prime of their life when struck with the devastating diagnosis of cancer may have different priorities to older adults. The establishment of a dedicated multidisciplinary adolescent and young adults oncology team that consists of physicians and allied health professionals with training and experience in addressing the needs of this unique set of patients, and incorporating fertility preservation as one of its pillars, is the way forward.

\section{References}

1. Peccatori FA, Azim HA Jr, Orecchia R, et al. Cancer, pregnancy and fertility: ESMO Clinical Practice Guidelines for diagnosis, treatment and follow-up. Ann Oncol 2013;24 Suppl 6:vi160-70.

2. Loren AW, Mangu PB, Beck LN, et al. Fertility preservation for patients with cancer: American Society of Clinical Oncology clinical practice guideline update. J Clin Oncol 2013;31:2500-10.

3. Chung JP, Haines CJ, Kong GW. Sperm cryopreservation for Chinese male cancer patients: a 17-year retrospective analysis in an assisted reproductive unit in Hong Kong. Hong Kong Med J 2013;19:525-30.

4. Rosen A, Rodriguez-Wallberg KA, Rosenzweig L. Psychosocial distress in young cancer survivors. Sem Oncol Nurs 2009;25:268-77.

5. Gorman JR, Bailey S, Pierce JP, Su HI. How do you feel about fertility and parenthood? The voices of young female cancer survivors. J Cancer Surviv 2012;6:200-9.

6. Canada AL, Schover LR. The psychosocial impact of interrupted childbearing in long-term female cancer survivors. Psychooncology 2012;21:134-43.

7. Partridge AH, Gelber S, Peppercorn J, et al. Web-based survey of fertility issues in young women with breast cancer. J Clin Oncol 2004;22:4174-83.

8. Ruddy KJ, Gelber SI, Tamimi RM, et al. Prospective study of fertility concerns and preservation strategies in young women with breast cancer. J Clin Oncol 2014;32:11516.

9. Senkus E, Gomez H, Dirix L, et al. Attitudes of young patients with breast cancer toward fertility loss related to adjuvant systemic therapies. EORTC study 10002 BIG 3-98. Psychooncology 2014;23:173-82.

10. Chung J, Lao T, Li T. Evaluation of the awareness of, attitude to, and knowledge about fertility preservation in cancer patients among clinical practitioners in Hong Kong. Hong Kong Med J 2017;23:556-61.

11. Lambertini M, Del Mastro L, Pescio MC, et al. Cancer and fertility preservation: international recommendations from an expert meeting. BMC Med 2016;14:1. 